\title{
Research of structural color of butterfly wings
}

\author{
Masha $\mathrm{Li}^{1, \mathrm{a}}$, Yang Wang, Caijin Zhang, Shu Yang ${ }^{* 1, b}$ \\ ${ }^{1}$ Nanhu College, Jiaxing University, Zhejiang, Jiaxing, 314000, China \\ a13456323962@163.com, byangshu5678@163.com
}

\begin{abstract}
Keywords: butterfly wings; scales; structural coloration; photonic crystal
Abstract: In order to achieve color fabric without dyeing, people dedicate to the research and development of green textiles. Structural coloration, also known as physical chromophore, with high saturation, high brightness, never fade, environmental friendly, By analyzing the structural coloration of the Mechanism and put forward the structure color instead of chromogenic dye. Through the observation of optical discoloration on the butterfly scales, one kind of photonic crystals, found that the optical effects make the scales show different colors.
\end{abstract}

\section{Introduction}

Many living organisms take on the color of the rainbow in nature. From the point of causing the coloration, the colors present in organisms are divided into two categories: chemical coloration and structural coloration [1]. The chemical coloration is arose from the pigment, besides not all colors come from the pigment, but the structural color, also called physical color, produced by the pure physical structure [2,3]. The structural coloration are usually possessed of gloss, and the color will make changes with different angles that commonly can be seen from butterfly wings, plumage, opal gemstone, seafood shells, beetle surface and so on [4].

Photonic crystal structure [5,6] is the one of mechanism accounts for the structural color. The well-known example among the structural coloration produced by photonic crystal is the sea mouse (Aphroditidae polychaeta) [7] living about the sea in Australia, the bristle of sea mouse has bright iridescent color.

The significance of studying the structural coloration in biology is to clarify the secret of structural colors, subsequently the method of bionics can be available to draw the theory of color indication into the design of color fabric, so that the color fabric without dying will come true. This paper study two species of common butterfly living in temperate to observe the different color positions on the scales, and proceed to analyze the chromatic features.

\section{The study of optical discoloration on the scale}

The paper select two kinds of butterflies living in temperate as subjects, are sasakia charonda and papilio maackii respectively. The sasakia charonda belongs to lepidoptera which is selected as the national butterfly in Japan. The papilio maackii is a large butterfly which belongs to the lepidoptera papilionidae.

Select two position of different colors in advance on each scale, shown as Fig.1(a) which indicates the two areas to be observed on wings of sasakia charonda, and the positions for observation on wings of papilio maackii is marked in Fig.1(b). Scanning electron microscope is adopted to make careful observation for the two sections of each sample. 


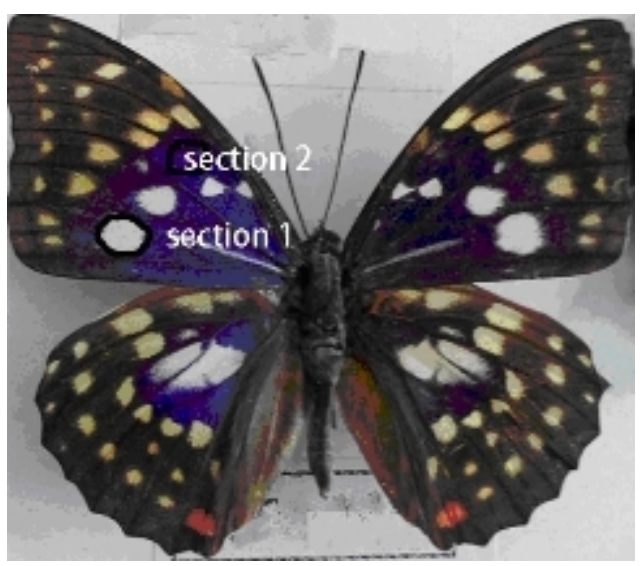

(a)

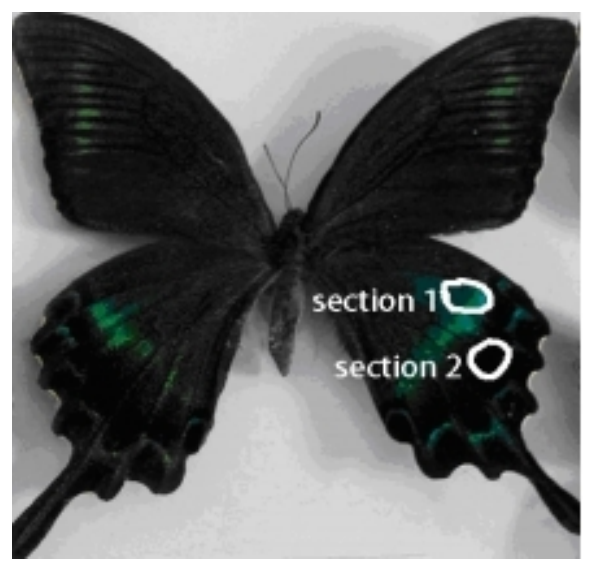

(b)

Fig.1 Two positions for observation on wings of (a) Sasakia charonda and (b) Papilio maackii

\section{Alter the packing medium}

The butterfly scale is of two-element crystal complex structure composed of chitin arranging in air according to periodic structure to some degree. The solid material of scale is chitin, the refractive index is of $1.56 \pm 0.01$, air with refractive index at 1.0 serves as packing medium. The study makes attempt to alter the packing medium to observe that if the scale shows different colors.

(1) The blue-purple areas of sasakia charonda

Fist select the scales of blue-purple areas of sasakia charonda as subjects, put the sample for observation on the objective table, drop in 1 to 2 drops of $75 \%$ ethanol with dropper on the surface of sample, shot the color image of scales before dropping 3 seconds after dropping, 10 seconds after dropping, 20 seconds after dropping, shown as Fig.2.

Found that 3 seconds after dropping the ethanol into the scale of sasakia charonda, it is the ethanol filled to the scale medium inter instead of air afterwards, the color of scale altered prominently, with the blue-purple turning into the red-brown, along with the alcohol volatilizing, namely at the 10 seconds, the red-brown become thinning gradually with the areas of blue-purple increasing, the scale return to the original color of blue-purple till 20 seconds when the evaporation of ethanol coming to an end.

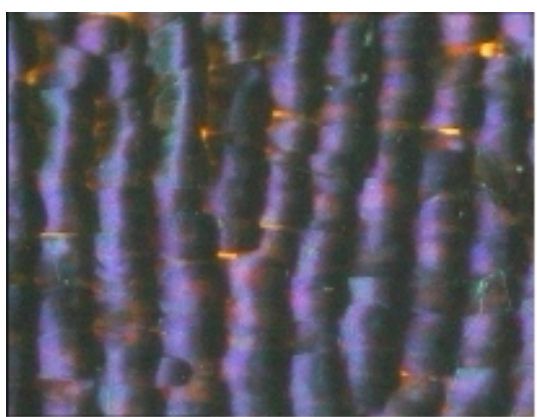

(a)

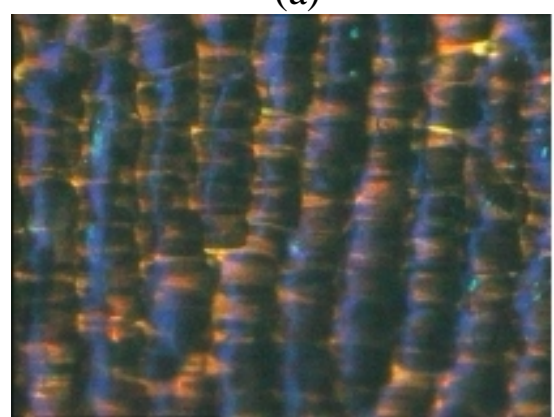

(c)

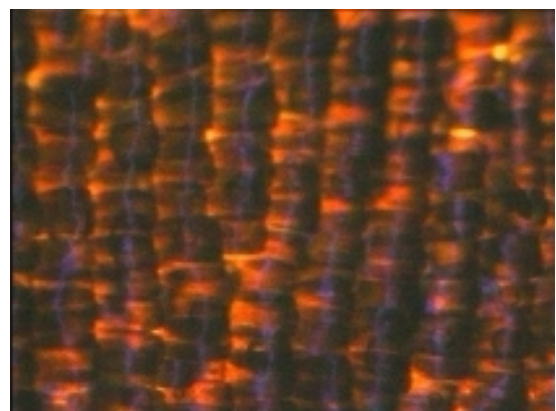

(b)

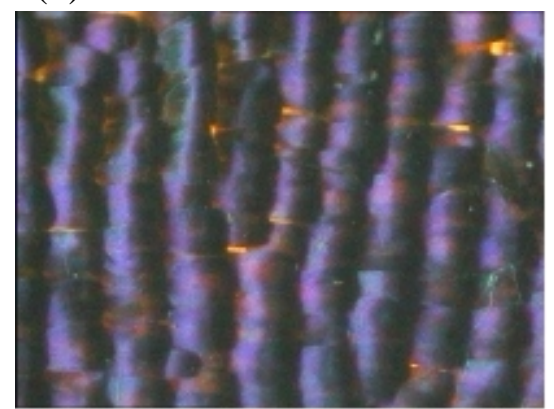

(d)

Fig. 2 Color of Sasakia charonda with dropping of ethanol(a)before dropping(b)3 seconds after dropping(c)10 seconds after dropping (d) 20 seconds after dropping 
(2) The green areas of papilio maackii

Next conduct the same experiment as above in the interest of the green areas of papilio maackii, the results shown as the Fig.3.

It can be found out , 3 seconds after dropping the ethanol into the scale of papilio maackii, ethanol filled to the scale medium inter instead of air afterwards, the color of scale alters prominently, with the bright green turning into the purple-black, along with the alcohol volatilizing, namely at the 10 seconds, the purple-black of the scale become thinning gradually, the bright green begins to appear, the scale return to the original color of bright green till 20 seconds when the evaporation of ethanol coming to an end.

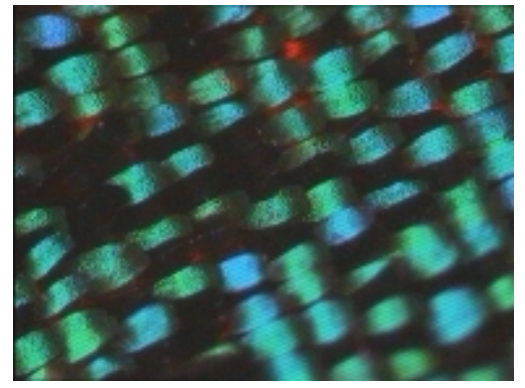

(a)

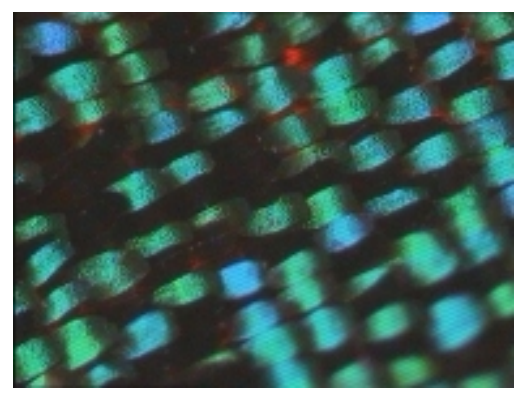

(c)

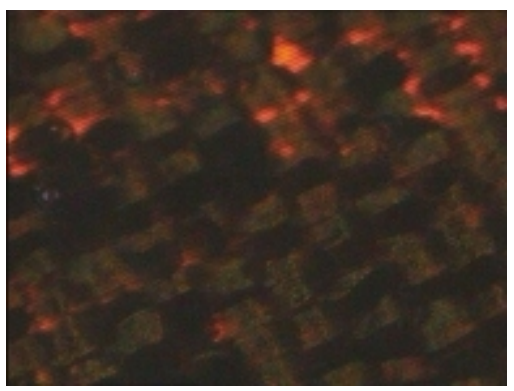

(b)

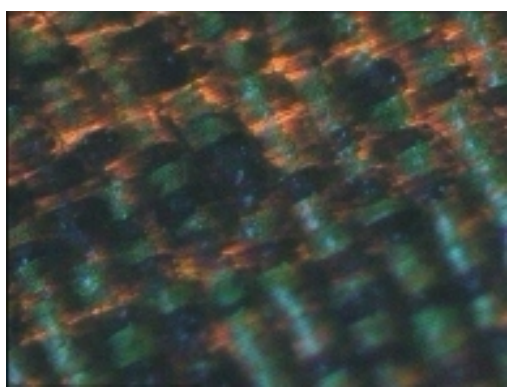

(d)

Fig. 3 Color of Papilio maackii with dropping of ethanol(a)before dropping(b)3 seconds after dropping(c)10 seconds after dropping (d) 20 seconds after dropping

It can be proved that the color of two butterfly scales is not caused by pigment going through the study, but due to the micro-structure of scale. Also can be inferred, it is the photonic band gap produced by photonic crystal comprises of chitin material and air that make an effect on the coloration formed by the transmission of lights.

Alter incident angle

The color of butterfly scale is not only affected by the transformation of dielectric refractive index, but also the diversification of light incident angle. The study altered the incident angles by means of incident light sources to observe the color transformation of scale.

Fig.4 (a) shows the vertical shot of incident light, it is visible that the green scale on the wing of butterfly partly appears to be emerald green, while Fig.4 (b) indicates the incident ray after spinning in 45 degrees, the color of the area on the butterfly scales transforms into blue-green, this study confirms that the color of butterfly scales is structural coloration, as well as dependent on angle. 


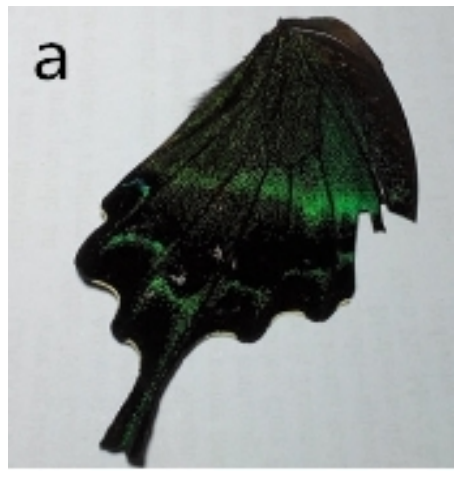

(a) vertical shot

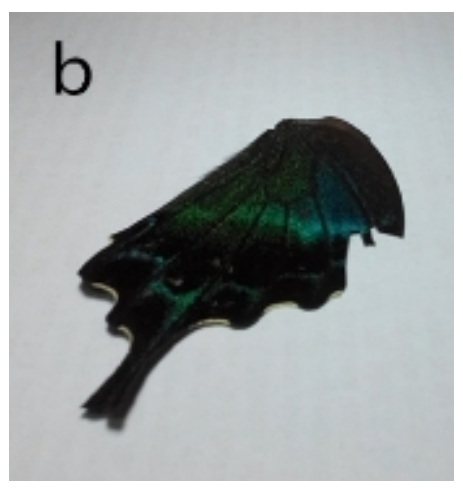

(b)shot in 45 degrees

Fig.4 Colors of Papilio maackii dependent on angles

\section{Conclusions}

The scale on the wing of butterfly is provided with complicated micro-structure, optical effect takes place as a result of photonic band gap when light shining on the scale, thus the gorgeous structural color come into being. Observation and research are in progress in the study aiming at the different color sections of two butterflies living in temperate zone, By way of altering the packing medium, it is approved that the formation of butterfly scales coloration is due to the photonic crystal structure, and the structural color has dependence. The research aiming at the specific appearance of color will precede in future study.

\section{Acknowledgement}

This work was financially supported by the National Natural Science Foundation of China (Grant No. 51403078), China Scholarship Council(Grant No.201508330017) and Zhejiang province education department(Grant No.Y201226223).

\section{References}

[1] Kinoshita, S. and Yoshioka, S. Structural Colors in Nature: The Role of Regularity and Irregularity in the Structute. Chemphyschem, (2005)6: 1442-1459.

[2] Kinoshita, S, Yoshioka S, Yasuhiro F, et al. Photophysics of structural color in the Morphobutterflies. Forma, 2002, 17: 103-121.

[3] Kinoshita S, Yoshioka S, Miyazaki J. Physics of structural colors. Rep Prog Phys, 2008,

[4] Parker, A.R. (1998) Colour in Burgess Shale animals and the effect of light on evolution in the Cambrian, Proc. R. Soc. Lond. B 265: 967-972.

[5] Yablonovitch E., Inhibited spontaneous emission in solid-state physics and electronics [J], Phys. Rev. Lett., 1987, 58, 2059.

[6] John S., Strong localization of photons in certain disordered dielectric superlattices [J], Phys. Rev. Lett., 1987, 58, 2486.

[7] Parker A. R., McPhedran R. C., McKenzie D. R., Botten L. C., Nicorovici N.- A. P., Photonic engineering-Aphrodite' iridescence [J], Nature, 2001, 409, 36. 